\title{
Wireless Sensor Network Technologies for Condition Monitoring of Industrial Assets
}

\author{
Spilios Giannoulis ${ }^{1}$, Christos Koulamas ${ }^{1}$, Christos Emmanouilidis ${ }^{2}$, \\ Petros Pistofidis ${ }^{2}$, and Dimitris Karampatzakis ${ }^{2}$ \\ "ATHENA" Research and Innovation Center in \\ Information, Communication and Knowledge Technologies \\ ${ }^{1}$ Industrial Systems Institute, Patras, Greece \\ \{sgiannoulis, koulamas\} @isi.gr \\ ${ }^{2}$ Institute for Language and Speech Processing, Xanthi, Greece \\ \{chrisem, pistofidis, karampatzakis\}@ceti.gr
}

\begin{abstract}
Systematic and robust condition monitoring of crucial equipment is the cornerstone of any successful preventive maintenance policy in the industrial environment. Recent advances in low-cost wireless sensor network (WSN) technologies and products indicate a promising future for a cost-effective, wider and more permanent deployment of a distributed sensing and processing infrastructure. This paper aims to provide a comprehensive assessment of main WSN technology alternatives available today, based on a qualitative and quantitative analysis of the typical range of requirements in the specific application domain of industrial machine condition monitoring.
\end{abstract}

Keywords: Industrial Wireless Sensor Networks, Asset Management.

\section{Introduction}

Over the last years numerous advancements have boost the use of wireless communications in many areas of the industry. Wireless communications have become standardized and can provide many options to the industry engineer to choose from, in order to design and deploy a wireless communication infrastructure. With speeds ranging from few Kbps to more than $100 \mathrm{Mbps}$ and the cost of a wireless node dropping every day, wireless technologies look even more appealing. Then the notion of wireless sensor networks (WSN's) came into play to provide the wireless node with intelligence and upgrade it from a typical routing/transmitting mechanism to an intelligent sensing device. Every node in a WSN can now control autonomously a set of transducers or composite sensors, execute complex data processing techniques to the sensor data and organize, support and manage distributed applications in cooperation with other nodes in the network.

The paper is structured as follows. In the first part, the focus is on the definition of the most important application specific characteristics, the requirements on the services that a WSN infrastructure has to provide, as well as the main trade-offs in the 
design process. In the second part, the dominant wireless communication technologies and standards are presented, pointing out the pros and cons against each requirement and trade-off axis based on the requirements of Industrial Asset Management applications. The goal of this paper is mainly to pinpoint the possible characteristics of each technology that provide specific advantages within the Application Domain and try to point in the direction of a technology that should exhibit most of these characteristics in the future.

\section{Domain Characteristics}

Looking into the wider industrial monitoring and control systems supporting the application domain of scope, the mostly cited efforts on addressing important specific requirements can be structured in: a) the real-time performance and dependability axis, and b) the seamless integration and interoperability axis.

Regarding the first axis, systems and networks needed for condition monitoring applications used in the context of a preventive maintenance policy implementation may be safely categorized under the SP100 classes 5 and/or 4, that is, logging, downloading/uploading and alerting systems. Typical periodicities of sampling simple, one-dimensional sizes, such as temperatures, pressures etc. do not introduce really strict real-time requirements, since the machine wear - which is the main monitoring objective - usually evolves in a wider time scope. However, more complex measurements such as vibration monitoring may pose stricter real-time requirements, when it comes to certain architectural and design decisions over the handling of the time series that constitutes one "sample". This depends on the needed size of the sample and the complexity of the applied algorithmic processing (e.g. for novelty detection and/or fault diagnosis), which, in turn, define alone a design space with certain decisions on computation / storage / bandwidth trade-offs. As an indicative order of magnitude, consider that a $16-\mathrm{bit} / 80 \mathrm{kHz}$ vibration sample produces $160 \mathrm{kByte} / \mathrm{sec}$ that needs to be handled. The approach of data preprocessing in the wireless node reduces the volume of data that need to be transmitted to the data server, however in some cases the raw sample data is required to be transferred, either for simple data logging purposes or for more complex data processing, leading to extensive networking transmission load and increased latency if the networking technology is not able to handle effectively the volume of the raw data set.

Furthermore, synchronization requirements may also exist between measurements at different sensing points when for example collaborative sensor applications are the case at hand. Adding into the picture the wireless nature, related to medium behavior aspects - especially in the noisy industrial environment - as well as to energy related constraints, it is also obvious that the trade-off space may drastically increase.

Finally, the demand for seamless integration and interoperability clearly indicates a necessary focus on established international standards for any large scale industrial investment to be justified. These standards mainly have to address the networking and inter-networking layer, as well as the application level protocols, data definition and architectural layers. Since in this paper the focus is on networking technologies, the 
main relevant standards and industrial specifications are presented and discussed in the following sections.

\section{WSN Technologies and Standards}

Three main standardized wireless technologies exist today that can be used in the context of WSN's to provide the required connectivity mechanisms. These include IEEE 802.11 [1], IEEE 802.15.1[2] and IEEE 802.15.4 [3]. Based on these standards several enhancements/related standards or products have been presented like the WirelessHART [7] and ISA 100.11a [8] standards that are based partially on the IEEE 802.15.4, as well as Bluetooth [5] and WISA [9], that are based fully or partially on IEEE 802.15.1 respectively. The characteristics of each standard/solution shall be presented in this section and the pros and cons of each protocol shall be exposed with respect to the application domain characteristics and requirements.

The IEEE $802.11 \mathrm{x}$ family of standards defines the physical and MAC layer protocols that can match perfectly to the design and organization of a wireless local area network (WLAN). Various transmission speeds are supported from 2 Mbps up to hundreds of Mbps with the latest 802.11n standard that introduces MIMO techniques to mitigate the effect of the multipath problem, as well as a range of transmission up to hundreds of meters outdoors and approximately 50-60m indoors. This technology can still be used in WSN's with the disadvantage of relatively high power consumption opposed to the low power requirements of a WSN. Even more, IEEE 802.11x family suffers from interference issues and exhibits reduced robustness especially in the presence of Bluetooth/IEEE 802.15.4 that both use the same ISM 2.4 GHz band as well as possible interference from industrial processes present in close proximity of the transceiver. The use of a CSMA based MAC algorithm provides non-deterministic timing behavior and therefore cannot support strict real time application requirements especially if the typical state of the network in terms of data load is anything else than low.

The IEEE 802.15.1 standard on the other hand came at first to fulfill the need for wireless connectivity in the range of a few meters, hence it was characterized as a personal area network protocol (PAN). Its main target was to replace wires connecting devices like keyboards and mice to a pc, speakers to any kind of sound source, and in general any low-range, low-bandwidth wired solution until that day. The idea of using IEEE 802.15.1 as a wireless solution to enable wireless networking came together with Bluetooth, an enhancement of IEEE 802.15.1 focusing mainly on upper layers of a wireless network providing advanced routing and application specific profiles as well as 3 classes of possible transmitting power that provide different communication range from few meters (class 3 device) up to $100 \mathrm{~m}$ maximum (class 1 device). The maximum supported theoretical throughput is $3 \mathrm{Mbps}$ in Bluetooth v2.0 standard although it has been found that in practice no more than $2.1 \mathrm{Mbps}$ can be achieved due to protocol overhead. For Bluetooth v1.x the maximum theoretical throughput is 1 Mbps but it has been shown in [11] that it cannot surpass the $700 \mathrm{kbps}$ barrier for the same reason as stated before, protocol overhead. The main 
disadvantage of IEEE802.15.1/Bluetooth, which hinders its wider adoption in WSN's, is its small range and restricted support of scalability since it supports only a few nodes per Piconet, the proposed cluster of devices in Bluetooth standard employing a single master-multiple slaves stations architecture. Its main advantage is its real time behavior based on the MAC layer used, as well as in the application profiles defined by Bluetooth for time critical applications.

The WISA solution that is based on IEEE 802.15.1 offers re-usability of wireless frequencies forming cells much like in the mobile telephony networks. Using TDMA as well in the MAC layer, WISA provides deterministic behavior as well as advanced interference deterrent techniques like frequency division duplex and frequency hopping to minimize interference in the industrial environment. It should also be noted that in addition to the wireless communication solution, WISA also provides a wireless power provisioning solution based on long-wave radio frequencies that takes advantage of the inductive magnetic coupling phenomenon. This technology could render WISA a completely wireless and free from power constraints solution for the industrial environment.

The IEEE 802.15.4 standard provides wireless communication speeds up to 250 kbps and communication range up to $60-80 \mathrm{~m}$ outdoors as well as low power characteristics that make it an ideal candidate for use in the context of wireless sensor networks. It can be considered a middle solution between IEEE 802.11 and IEEE 802.15.1 standards in terms of range but not in terms of power and throughput, since it exhibits the lowest power consumption making it ideal for WSN but also the lowest link throughput. Based on the IEEE 802.15.4, several standards exist that focus on providing higher layer services like routing, application profiles etc. These standards include ZigBee [4]/ZigBee Pro, an analogous of what Bluetooth is for IEEE 802.15.1, as well several other standards that try to provide enhanced services like the 6LowPAN standard from IETF.

ZigBee provides on top of the IEEE 802.15.4 PHY and MAC layers, the Network and Application layer as well as security services. The most important characteristic that ZigBee adds is the mesh networking capabilities combining Tree Routing with on demand nonTree (mesh) Routing based on the well-known AODV routing algorithm [13]. It also natively supports both star and tree typical networks topologies. Besides adding two high-level network layers to the underlying structure, another significant improvement is the introduction of ZigBee Device Objects (ZDOs). These are responsible for a number of tasks, which include keeping of device roles, management of requests to join a network, device discovery and security. Compared to IEEE 802.15.4 it improves greatly the possible selection of topologies to enable the deployment of a ZigBee based network as well as adding security mechanism on top of IEEE 802.15.4. and vendor specific ZDOs to enable interoperability between different vendor devices. ZigBee Pro enhances transmission range up to hundreds of meters by increasing the transmission power and receiver sensitivity.

6LowPAN enables the use of IPv6 addressing over IEEE 802.15.4 based WSN's hence enabling the Internet of Things notion where every node in a WSN is visible as a device from any connected IP based network machine transparently. Its main advantages are the reuse and interoperability of already known and tested services and 
application data models like HTTP, HTML and XML as well as the transport subprotocols of IP, TCP and UDP. Furthermore, all existing IP based security and network management infrastructure already established in Industrial Environments can be used with minimal integration efforts.

WirelessHART on the other hand focuses in providing interoperability with wired Hart networks. Using frequency hopping combined with the DSSS modulation of the 802.15.4 radios, it increases the resistance of the WSN to common interference sources such as $802.11 \mathrm{x}$ when operating in the $2.4 \mathrm{GHz}$ band, and Bluetooth as well as several industrial processes and devices. Also the use of TDMA instead of CSMA/CA MAC algorithm as defined in IEEE 802.15.4, results in deterministic behavior of the WirelessHART in the time domain. Finally, the ISA 100.11a standard is also based on the IEEE 802.15.4 PHY. It also adds support for TDMA like WirelessHART, but also supports Channel Hopping and Mesh Routing in the lower layers while providing reliable and secure operation for critical monitoring, alerting, supervisory control, open loop and closed loop control applications.

\section{Comparing the Alternatives}

IEEE $802.11 x$ family of standards offers high bandwidth that may be needed for continuous data logging purposes but suffers from interference from other devices in the 2.4 GHz ISM band as well as from typical industrial operations. The use of a CSMA based algorithm in MAC layer results in non-deterministic timing behavior, hindering the usage of IEEE 802.11x in time critical applications. The fact that it supports high bandwidth modes of operation lessens the impact of the CSMA algorithm when low network data load is the case. Its wide transmission range provides efficient and less challenging deployment methodologies to be used with few nodes being able to provide coverage to a large industrial area. This characteristic is also the reason that IEEE $802.11 \mathrm{x}$ is not able to provide a dense network solution for WSN's, since its wide range and the CSMA MAC algorithm used are a poor combination when dense wireless networking topologies is one of the requirements. It must be also noted that the maximum throughput obtained most of the times is far from the theoretical optimal especially if the wireless data load is high, due to CSMA based MAC, and also if there is interference of any kind.

IEEE 802.15.1 offers low bandwidth, low transmission range, does not scale well, but offers good real time support at all times. This is mainly due to the TDMA MAC algorithm used, as well as because of the requirement imposed from the standard that only 7 active slave nodes can coexist per master node at all times. This behavior results in IEEE 802.15.1/Bluetooth to be a good candidate for alarm/condition monitoring in critical industrial systems where conditions change frequently and in a matter of seconds. On the other hand it is not suitable to be used for data logging/continuous data transfer since the bandwidth offered is low and cannot fulfill the needs imposed by the data logging application.

WISA provides deterministic behavior by using a TDMA based MAC algorithm, further optimized to provide up to 120 sensors/actuators per Base Station as well as 
short cycle times up to $2048 \mu$ s. Furthermore, it adds advanced interference mitigation techniques to minimize interference in the harsh industrial environment and there is no need for frequency planning in case of multiple cells since the FH sequences are constructed to guarantee adequate separation between consecutive hops and low correlation. In all cases, all operating cells use the entire available ISM band of $80 \mathrm{MHz}$ at $2.2 \mathrm{GHz}$ [9].

The IEEE 802.15.4 standard offers medium transmission range, medium bandwidth, and good scalability but suffers from time characteristics due to the CSMA based MAC layer used in IEEE802.15.4 and all standards based on it like ZigBee and ZigBee Pro. Only ZigBee Pro supports communication ranges up to few hundreds of meters duo to increased transmission power that results in lower power aware characteristics. As it was shown in [12], IEEE 802.15.4 performance is substantially lower in terms of throughput in comparison with the throughput defined in the standard. In fact no more than $140 \mathrm{Kbps}$ can be achieved in any case when the standard states that the theoretical speed of IEEE 802.15 .4 is $250 \mathrm{Kbps}$. This difference is based mainly in the small packet size defined in the standard that in conjunction with the required protocol data overhead present in each packet results in a $44 \%$ reduction of actual application data throughput.

ISA100.11a and WirelessHART on the other hand provide real time characteristics since they are based on a TDMA MAC as well as robust operation, increasing the resistance of the WSN to common interference sources by the usage of frequency/channel hopping combined with the DSSS modulation. The above characteristics result in proclaiming ISA100.11a and WirelessHART better candidates than standard IEEE802.15.4/ZigBee/ZigBee Pro, while the main disadvantage is the lack of wide availability of products, so far.

Comparing all families of standards, it seems that IEEE 802.15.4 provides today a middle-level solution capable to support the wider possible range of related trade-offs, excluding the extreme cases of high-bandwidth, continuous data logging scenarios. Combined with 6LoWPan it may also offer superior interoperability and scalability since it provides IPv6 addressing and so direct communication with IP based backbone infrastructures, eliminating the usage of application level gateways and protocol translators. It should also be noted that in general, low power wireless solutions like IEEE 802.15.4/ZigBee and /IEEE 802.15.1/Bluetooth are well suited for low data rate industrial applications duo to their low power characteristics that result in maximized longevity of the provided solution. But for high data rate applications, solutions like the IEEE $802.11 \mathrm{x}$ should be considered since they offer lower normalized power consumption per transmitted byte compared to IEEE 802.15.1 and IEEE 802.15.4 as it has been shown in [10].

In Table 1, a qualitative comparison of the aforementioned protocols and products is presented with respect to the requirements imposed by the applications of the Condition Monitoring for Industrial Assets domain. 
Table 1. Comparison of the presented Wireless Communication Standards based on the requirements of Industrial Asset Management

\begin{tabular}{ccccccc}
\hline $\begin{array}{c}\text { Wireless } \\
\text { Technology }\end{array}$ & Robustness & $\begin{array}{c}\text { Real Time } \\
\text { Performance }\end{array}$ & Range & $\begin{array}{c}\text { Link } \\
\text { Throughput }\end{array}$ & $\begin{array}{c}\text { Network } \\
\text { Scalability }\end{array}$ & $\begin{array}{c}\text { Power } \\
\text { awareness }\end{array}$ \\
\hline IEEE 802.11 & $*$ & $*$ & $* * *$ & $* * *$ & $* *$ & $*$ \\
IEEE 802.15.4 & $* *$ & $*$ & $* *$ & $*$ & $* *$ & $* * *$ \\
ZigBee & $* *$ & $* *$ & $* *$ & $*$ & $* * *$ & $* * *$ \\
ZigBee Pro & $* *$ & $* *$ & $* * *$ & $*$ & $* * *$ & $* *$ \\
IEEE 802.15.1 & $* *$ & $* * *$ & $*$ & $* *$ & $*$ & $* *$ \\
Bluetooth & $* *$ & $* * *$ & $*$ & $* *$ & $*$ & $* *$ \\
WirelessHART & $* * *$ & $* * *$ & $* *$ & $*$ & $* *$ & $* * *$ \\
ISA 100.11a & $* * *$ & $* * *$ & $* *$ & $*$ & $* *$ & $* * *$ \\
Bluetooth & $* *$ & $* * *$ & $*$ & $* *$ & $*$ & $* *$ \\
WISA & $* * *$ & $* * *$ & $*$ & $* *$ & $* *$ & $* * *$ \\
\hline
\end{tabular}

\section{Conclusion}

This paper presents the requirements imposed by the applications of Industrial Asset Management and Preventive Maintenance. It then focuses on the standardized wireless technologies available today and presents their advantages and disadvantages resulting in a qualitative assessment of each wireless technology with respect to the requirements of Industrial Asset Management applications. It is evident that each technology has its specific advantages and disadvantages and can be used for specific applications of Asset Management but no one-fits-all solution exists at this time. As a final conclusion, it seems that in a future wireless standardization effort, several advantages could and should coexist like IP based communication, physical layer robustness due to the use of frequency division duplex and/or frequency hopping as well as possible MIMO antenna techniques to reduce the multipath effect. Last but not least TDMA based MAC protocol could be supported to provide real time performance which is so critical for most Industrial Applications. In this context, the two standards focusing in Industrial environment applications, the WirelessHart and ISA100.1a seem promising and it is our intention to evaluate them through extended benchmarks and QoS testing when several of-the-self products become available in order to have a detailed view of their behavior and QoS performance.

Acknowledgements. The financial support received through GSRT (grant 09SYN71-856, project WelCOM) is gratefully acknowledged.

\section{References}

1. IEEE 802.11 standard, http://standards. ieee.org/about/get/802/802.11.html 
2. IEEE Std 802. Part 15.1: Wireless Medium Access Control (MAC) and Physical Layer (PHY) Specifications for Wireless Personal Area Networks (WPANs). IEEE Standards Association (January 15, 2005) ISBN 0-7381-4707-9, doi:10.1109/IEEESTD.2005.96290

3. IEEE Std 802. IEEE Standard for Information technology - Telecommunications and information exchange between systems - Local and metropolitan area networks - Specific requirements - Part 15.4: Wireless Medium Access Control (MAC) and Physical Layer (PHY) Specifications for Low-Rate Wireless Personal Area Networks (WPANs) (April 15, 2006)

4. ZigBee specification, Zigbee alliance (2008), http: / / www . zigbee. org

5. Bluetooth, http://www.bluetooth. com

6. http://datatracker.ietf.org/wg/6lowpan/

7. IEC 62591 ed1.0 - Industrial communication networks - Wireless communication network and communication profiles - WirelessHART ${ }^{\mathrm{TM}}$

8. ISA-100.11a-2011 - Wireless systems for industrial automation: Process control and related applications

9. Scheible, G., Dzung, D., Endresen, J., Frey, J.-E.: Unplugged but connected - Design and Implementation of a Truly Wireless Real-Time Sensor/Actuator Interface. IEEE Ind. Electron. Mag. 1(2), 25-34 (Summer 2007)

10. Lee, J.-S., Su, Y.-W., Shen, C.-C.: A Comparative Study of Wireless Protocols: Bluetooth, UWB, ZigBee, and Wi-Fi. In: Proceedings of the Industrial Electronics Society (IECON 2007), November 5-8, pp. 46-51 (2007)

11. Valenti, M.C., Robert, M., Reed, J.H.: On the throughput of Bluetooth data transmissions. In: IEEE Wireless Communications and Networking Conference (WCNC 2002), March 17-21, vol. 1, pp. 119-123 (2002), doi:10.1109/WCNC.2002.993475

12. Sun, T., Chen, L.-J., Han, C.-C., Yang, G.: Measuring effective capacity of IEEE 802.15.4 beaconless mode. In: IEEE Wireless Communications and Networking Conference (WCNC 2006), April 3-6, vol. 1, pp. 493-498 (2006), doi:10.1109/WCNC.2006.1683513

13. Perkins, C.E., Royer, E.M.: Ad-hoc on-demand distance vector routing. In: Proceedings of the Second IEEE Workshop on Mobile Computing Systems and Applications, WMCSA 1999, February 25-26, pp. 90-100 (1999), doi:10.1109/MCSA.1999.749281 